\title{
Prevention of Implications of Poor Posture and Physical Inactivity among Dentists
}

Long sitting hours, sedentary lifestyle has taken a toll on the well-being of the species. Studies have time and again reported that posture related problems in dentists has shown a very high prevalence. As the art of dentistry requires a lot of precision and patience, it can cause a plethora muscle and back-related problems. Most of these musculoskeletal problems are confined to the head and neck region and are usually due to long hours put in by the dentist while treating his patients. A Bad posture during the dental procedures put the dentist at risk for various dental procedures, however, it has been noted that there is lack of awareness about the implications of bad posture and physical inactivity amongst the dentists. This review is on the prevention and implications of poor posture and physical inactivity among dentists.

\section{INTRODUCTION}

Long sitting hours, sedentary lifestyle has taken a toll on the well-being of the species. The prevalence of posture related problems in dentists is very high. Dentistry requires a lot of precision, patience and can be back-breaking. With increasing awareness, the number of dental patients are increasing and so is the chair time for a dentist. Most of these musculoskeletal problems are confined to the head and neck region. Bad posture during the dental procedures is implicitly a risk factor in the increasing prevalence of musculoskeletal problems in dental personnel. A study revealed that among 359 dentists only 6o dentists had no discomfort. It was found that $70 \%$ had pain and discomfort from either neck, shoulders or headaches. Major risk factors associated with these pains in dentists are repetitive movements of the trunk which are unidirectional, long, tedious static positions with no movement, uncomfortable work posture, less flexibility. ${ }^{1-3}$

A good posture provides higher working energy, comfort, ease of operation, less pain and muscular tension and reduced errors in the procedure. Whereas a bad posture may instigate fatigue, stress, negative work attitude, less precision in the procedures, reduced quality, higher incidence of musculoskeletal disorders. ${ }^{4}$

Dentists start working with the ideal postures and positions but overtime adopt different postures is surprising to know that even an ill-designed working stations drives the dentist to adopt different postures. $^{5}$

The relationship between static muscles contractions and muscle ischemia has been proven. The weaker muscles lead to a bad posture as they try to adapt by lengthening or shorting which may result in a muscle imbalance leading to pain and damage. ${ }^{3}$

Micro trauma caused by repetitive forces leads to inflammation and swelling The muscle also requires adequate recovery time, in dentistry mostly the operator has less periods of rest leading to less recovery and more damage. ${ }^{3}$ In a study was Navah Z. et al, there was a significant correlation between the time spent in sitting and the severity of LBA in dentists which was attributed to awkward postures.

Auxiliary equipment such as magnification systems also play a pivotal role in reducing the incidence of musculoskeletal problems as they help preventing bending of the head but can also lead to musculoskeletal problems if not used correctly.

It is of paramount importance that the dentist pays special attention to his posture and exercise regime to prevent the development of musculoskeletal problems. Some tips for the same are:

- Have a balance in the active and passive posture.

- To avoid long appointments or to take small breaks in between to walk.

- To take walking breaks in between appointments. 
- Perform stretching after every appointment.

- It is recommended to change posture and positions after a maximum for 1 hour. In case of long procedures, it is acceptable to take a 5minute break after 1 hour of operating to overcome the chances of developing posture related disorders. ${ }^{6}$

Exercises for the fitness of the dentists are divided majorly into two types- the aerobic exercise and stretching which can be performed independently.

Any exercise which gets blood pumping and large muscle group working is termed as Aerobic exercise. Swimming, brisk-walking, heavy cleaning, running, cycling are some examples of aerobic exercises. It has plethora of benefits including improved cardiovascular health, regulation of blood sugar, reduction of chronic pains, aids in sleep, enhancing brain power, boosts immune system. Decreased flow of oxygen to muscles is a major contributing factor in musculoskeletal disorders and aerobic exercise helps combat this.

An exercise regime should comprise of a warm up prior to the exercise and a cool down session post workout. The intensity of the workout should be monitored as well, the heart rate should reach $70 \%$ HR max. This can vary from person to person. It can be of 20-30-minute duration and about 3-4 times a week.

A balanced position can counteract the effects of prolonged sitting. The postural symmetry states that all the body horizontal lines including the eyes, shoulders, elbows, hips and knees horizontals should be parallel and perpendicular to the median line of the body.

A balanced posture allows the dentist to freely move and still have no harmful effects on the body. Stretching in between in the appointments has a remarkable role in preventing musculoskeletal diseases.

Following are some stretches that can be performed $^{7}$ :-

- A straight back and no slouching of shoulders.

- Inclining forward of maximum of 20 degrees, tilting to the side and rotations should be avoided.
- Placing the arms parallel to the body, forward orientation $=10$ degrees, fore arms raised 25 degrees from the horizontal.

- The angle between the thighs and shanks of 105$110^{\circ}$ or more.

- The thighs apart up to $45^{\circ}$, avoiding a rigid fixation of the hip joint.

- The feet on the floor oriented forwards in the same plane with the shanks; when the feet are symmetrically positioned below the operator hands, the posture is balanced.

Tucked chin: This stretch involves tucking the chin towards the throat until a stretch is felt at the posterior hair line, but be cautious not to bend the neck too far. Also apply gentle pressure in the occipital region to tip the head.

Scalene: This stretch is simple and can be performed sitting as well. Hold the side of the chair with one hand and use the other hand to tilt the neck in the other direction and rotate to the same side.

Pectoralis major stretch: This is performed in a standing position facing the corner of a wall about 2 feet away. Raise your arms against the wall and lean on the wall forward from the ankles with slightly bent knees. This stretch is felt on the front of the shoulders.

Hand stretch: Raise the arm forwards with palms facing outwards and finger spread and use the other hand to bend the wrist until a stretch is felt.

Lateral trunk stretching: This is a simple stretch that can be performed while sitting on a chair. Bend to one side with opposite side hand raised above the head.

Tensor Fascia Lata Stretch: This is a muscle of the thigh. To perform the stretch, stand near a wall and use the support the same side arm. The leg near the wall side is crossed behind the other leg. To fully stretch, bend the trunk away from the wall. It is felt under the thigh and knee.

Hamstring Stretching: While sitting fully back on the chair, extend one leg forward and slide the hands towards your foot as far as you can go and slowly release back to the starting position and 
repeat for the other leg.

Calf stretch: Stand on an inclined surface and bent forward with spine straight.

\section{CONCLUSION}

There is lack of awareness about the implications of bad posture and lack of physical activity amongst the dentists. More awareness should be spread regarding the same, during the graduation days also the professors should keep a strict check of the posture and emphasise on the importance of correct posture. A dentist needs to take care of his health and reassess his posture as well as his workstation. The work station of a dentist should to be tailor-fit for him/her so allow free movements without any deleterious effects. These habits can help every dentist in the long run for not only a better professional life but also a longer one.

\section{REFERENCES}

1. Hayes M, Cockrell D, Smith DR. A systematic review of musculoskeletal disorders among dental professionals. Int J Dent Hyg. 2009;7(3):159-65.
2. Navah ZR, Tal Y, Alona M, Tamar K. Musculoskeletal symptoms among dentists in relation to work posture. WORK: A Journal of Prevention, Assessment and Rehabilitation. 2000;15(3):153-8.

3. Bethany V, Keith V. Mechanisms leading to musculoskeletal disorders in dentistry. JADA. 2003;134:1344-50.

4. An introduction to ergonomics: risk factors, MSDs, approaches and interventions, A report of the Ergonomics and Disability Support Advisory Committee (EDSAC) to Council on Dental Practice (CDP). ADA.org. 2004. [Last Accessed 15 ${ }^{\text {th }}$ March, 2018]

5. Todd AI, Bennett AI. Physical implications of prolonged sitting in a confined posture - A literature review. Ergonomics SA. 2007;19:7-21.

6. Murphy DC. Ergonomics and The Dental Care Worker. American Public Health Associations. 1988.

7. Carolyn K, Allen CL. New Delhi: Jaypee Brothers; 1996. Therapeutic exercise Foundations and Techniques. $1^{\text {st }}$ ed.
Cite this article as:

Gupta A, Gupta S. Prevention of Implications of Poor Posture and Physical Inactivity among Dentists. Int Healthcare Res J 2018;2(6):128-130. doi: 10.26440/IHRJ/02_06/194

\section{AUTHOR AFFILIATIONS:}

BDS, MDS, Conservative Dentist and Endodontist, Private Practitioner

2. BDS, MDS, Prosthodontist and Implantologist, Private Practitioner

\section{Corresponding Author:}

Dr. Sudha Gupta (MDS)

Tooth Care Dental Clinic

\#219, Sector -10 Panchkula
For article enquiry/author contact details, e-mail at: manuscriptenquiry.ihrj@gmail.com 